\title{
CORRIGENDUM
}

\section{Cytosine accumulation as a measure of the proton electrochemical gradient acting on the overexpressed cytosine permease of Saccharomyces cerevisiae}

\author{
A. A. Eddy and P. Hopkins
}

Microbiology (1996), 142, 449-457

p. 451 , there were errors in the equation defining parameter $B$ in the solution for the steady-state value of the intracellular substrate concentration. The correct equations should read:

$A S_{i}^{2}+B S_{i}+C=0$

Here

$$
\begin{aligned}
A= & \Theta H_{\mathrm{i}}\left(2\left(1+\Theta^{2}\right) S_{\mathrm{o}}+(1+\Theta)^{2} K_{\mathrm{m}}\right) \\
B= & \frac{\Theta}{1+\Theta^{2}}\left[\left(\Theta(1+\Theta) K_{\mathrm{m}} H_{\mathrm{o}}-\left(1+\Theta^{2}\right) H_{\mathrm{i}} S_{\mathrm{o}}\right)\right. \\
& \times\left(\left(1+\Theta^{2}\right) S_{\mathrm{o}}+(1+\Theta) K_{\mathrm{m}}\right) \\
& \left.+\left(S_{\mathrm{o}}\left(1+\Theta^{2}\right)+\Theta(1+\Theta) K_{\mathrm{m}}\right)\left(\Theta^{2}(1+\Theta) K_{\mathrm{m}} H_{\mathrm{o}}-\left(1+\Theta^{2}\right) H_{\mathrm{i}} S_{\mathrm{o}}\right)\right] \\
& +(1+\Theta)^{2} \frac{V_{\mathrm{m}}}{L} K_{\mathrm{m}} H_{\mathrm{i}} \\
C= & -\Theta^{2}(1+\Theta)^{2} K_{\mathrm{m}} H_{\mathrm{o}} S_{\mathrm{o}}\left(S_{\mathrm{o}}+\frac{V_{\mathrm{m}}}{L}+K_{\mathrm{m}}\right)
\end{aligned}
$$

